\title{
Dissociable Effects of Lidocaine Inactivation of the Rostral and Caudal Basolateral Amygdala on the Maintenance and Reinstatement of Cocaine-Seeking Behavior in Rats
}

\author{
Kathleen M. Kantak, ${ }^{1}$ Yolanda Black, ${ }^{1}$ Eric Valencia, ${ }^{1}$ Kristen Green-Jordan, ${ }^{1}$ and Howard B. Eichenbaum ${ }^{2}$ \\ 1 Laboratory of Behavioral Neuroscience and ${ }^{2}$ Cognitive Neurobiology Laboratory, Department of Psychology, Boston \\ University, Boston, Massachusetts 02215
}

Cocaine addiction is a chronically relapsing brain disease, but its neural basis is not yet well understood. Clinical reports underscore the possible importance of associative processes for regulating at least some aspects of cocaine addiction. The present study reports the effects of reversible lidocaine-induced inactivation of rostral basolateral amygdala (rBLA) and caudal basolateral amygdala (CBLA) regions on the maintenance and reinstatement of drug-seeking behavior in rats trained to selfadminister $1 \mathrm{mg} / \mathrm{kg}$ cocaine under a second order schedule of drug delivery. Both regions of the basolateral amygdala were investigated because they have dissociable effects on cognitive task performance. Results demonstrated that after selfadministration training and a period of extinction and abstinence, lidocaine inactivation of the rBLA and CBLA attenuated the reinstatement of drug-seeking behavior induced by cocaine-associated cues examined in conjunction with a single priming injection of cocaine. In contrast, lidocaine inactivation of only the rBLA blocked reinstatement of drug-seeking behavior induced by cocaine-associated cues examined alone. Additional differences were shown during cocaine maintenance testing where inactivation of only the CBLA attenuated drugseeking behavior. Drug intake was not altered. Thus, the rBLA and $\mathrm{CBLA}$ appear to selectively and dissociably regulate drugseeking behavior under conditions of cocaine abstinence (cue-induced reinstatement) and repeated cocaine use (maintenance), respectively. These findings suggest that the basolateral amygdala may be more functionally heterogeneous than commonly thought for regulating drug-seeking behavior. The basis for this dissociation might be related to neuroanatomical connections of the rBLA and CBLA with segregated, but parallel, corticostriatalpallidothalamic circuits.

Key words: caudal basolateral amygdala; cocaine; drug cues; drug prime; lidocaine; maintenance; reinstatement; rostral basolateral amygdala
Cocaine addiction is a chronically relapsing brain disease. Clinical reports suggesting a link between limbic and cortical structures in mediating drug use and craving underscore the possible importance of associative processes for regulating some aspects of drug addiction. Imaging studies demonstrate that craving induced by cocaine-associated cues in abstinent addicts produces specific changes in patterns of activation in the amygdala, anterior cingulate, basal ganglia, dorsolateral prefrontal cortex, and cerebellum (Grant et al., 1996; Maas et al., 1998; Childress et al., 1999; Wang et al., 1999). Furthermore, Breiter et al. (1997) showed that craving and rush induced by a cocaine priming injection also modulate patterns of activation in memory circuits important for regulating cognitive functions. Animal studies examining cognitive aspects of addiction-related behavior are emerging, particularly in reference to the basolateral amygdala.

An associative conditioning process involving mechanisms within the basolateral amygdala is thought to underlie the salience of cues associated with cocaine self-administration, which induce drug-seeking behavior in laboratory animals (Whitelaw et al., 1996; Meil and See, 1997; Grim and See, 2000; Weiss et al.,

Received July 13, 2001; revised Nov. 9, 2001; accepted Nov. 14, 2001.

This research was supported by National Institute on Drug Abuse Grant DA11716 (K.M.K.) and National Institute on Mental Health Senior Scientist Award MH01475 (H.B.E.). We thank Katrina Gibson and Francisco Ugalde for technical assistance.

Correspondence should be addressed to Kathleen M. Kantak, Department of Psychology, Boston University, 64 Cummington Street, Boston, MA 02215. E-mail: kkantak@bu.edu.

Copyright (C) 2002 Society for Neuroscience $\quad 0270-6474 / 02 / 221126-11 \$ 15.00 / 0$
2000). Earlier lesion studies demonstrated that conditioned stimulus-reward association is dependent on an intact basolateral amygdala (Cador et al., 1989; Everitt et al., 1991). Recently, we reported that lidocaine-induced inactivation of the rostral basolateral amygdala (rBLA) and caudal basolateral amygdala (cBLA) produced dissociable effects on cognitive performance (Kantak et al., 2001). These findings suggest functional heterogeneity between the rBLA and cBLA for processing associative aspects of reward-relevant behavior. In support of this view, neuroanatomical studies show that rBLA and cBLA projection neurons are topographically organized and innervate distinct compartments within the nucleus accumbens core and shell (Gröenewegen et al., 1990, 1991). The core and shell, in turn, have dissociable control over reward-relevant behavior (Parkinson et al., 1999; Ito et al., 2000). Therefore, our cognitively identified sites in the rBLA and cBLA were manipulated to determine possible dissociable roles in regulating selfadministration behavior.

We used a model of maintenance and reinstatement that relies on second-order scheduling of drug and conditioned stimulus cues for maintaining high rates of performance (Spealman et al., 1999). With a second-order schedule, drug delivery follows completion of a fixed number of responses after a specified time interval elapses (drug-taking behavior). During intervals when drug is not immediately available, responses are maintained by presentation of a brief stimulus light previously paired with drug delivery (drug-seeking behavior). The advantage of using a second-order schedule is that drug delivery does not depend on 
the number of responses made during the preceding interval. Thus, drug intake is independent of the magnitude of drugseeking behavior (Goldberg et al., 1981; Kantak et al., 2000; Ito et al., 2000). It is suggested that second-order schedules of drug delivery provide a reliable means of investigating possible dissociable neurobiological mechanisms underlying drug-seeking and drug-taking behavior (Everitt and Robbins, 2000). Differentiation between drug-seeking and drug-taking is important because for humans who are addicted to drugs, the actions involved in drug acquisition differ from those required to take the drug (Olmstead et al. 2000).

\section{MATERIALS AND METHODS}

\section{Subjects}

Male Crl(WI)BR rats (Wistar rats; Charles River Breeding Labs, Portage, MI) were housed in individual hanging plastic cages $(24 \times 22 \times 20$ $\mathrm{cm})$ within a temperature- $\left(21-23^{\circ} \mathrm{C}\right)$ and light-controlled (on at 8:00 A.M.; off at 8:00 P.M.) vivarium. Between experimental sessions, the rats had continuous access to water in their home cages. To maintain body weight at $\sim 85 \%$ of adjusted ad libitum values throughout the duration of the study (from $338 \pm 7$ to $417 \pm 7 \mathrm{gm}$ ), $\sim 16 \mathrm{gm}$ of food was provided each day. The policies and procedures set forth in the National Institutes of Health Guide for the Care and Use of Laboratory Animals were followed.

\section{Apparatus}

Eight experimental chambers (model ENV-008CT; Med Associates, East Fairfield, VT) were each equipped with two response levers positioned $8 \mathrm{~cm}$ to the left and right of a center mounted food receptacle. Connected to the food receptacle was a pellet dispenser, which delivered $45 \mathrm{mg}$ food pellets (Traditional Formula; Noyes, Lancaster, NH). A white stimulus light was mounted $7 \mathrm{~cm}$ above each lever. Each chamber was outfitted with a single channel fluid swivel and spring leash assembly that were connected to a counterbalanced arm assembly (Med Associates). A sound-attenuating cubicle (model ENV-018 M; Med Associates) equipped with an overhead light to provide general illumination, a fan to provide ventilation, and an $8 \Omega$ speaker to provide sound stimuli enclosed each chamber. Motor-driven syringe pumps (model PHM-100; Med Associates) were used for drug delivery and were located outside the sound-attenuating cubicle. A 486 AT-compatible computer programmed in Medstate Notation and connected to an interface (Med Associates) controlled experimental events.

\section{Drugs}

The drugs used were cocaine hydrochloride (gift from the National Institute on Drug Abuse, Bethesda, MD) and lidocaine hydrochloride (Sigma, St. Louis, MO). Cocaine was dissolved in a sterile $0.9 \%$ saline solution containing $3 \mathrm{IU}$ of heparin $/ \mathrm{ml}$. Intravenous delivery of the 1.0 $\mathrm{mg} / \mathrm{kg}$ cocaine training dose was accomplished by infusing a $2.67 \mathrm{mg} / \mathrm{ml}$ solution though a $20 \mathrm{cc}$ filtered plastic syringe at a rate of $1.8 \mathrm{ml} / \mathrm{min}$. To attain a dose of $1.0 \mathrm{mg} / \mathrm{kg}$, inf usion volume was adjusted for body weight, resulting in drug delivery times of $\sim 4-5 \mathrm{sec}$ in individual rats. The heparinized saline solution was used during saline substitution aspects of the study. Lidocaine, which was infused intracerebroventricularly, was dissolved in sterile $0.9 \%$ saline to make $2 \%(20 \mathrm{mg} / \mathrm{ml}), 6 \%(60 \mathrm{mg} / \mathrm{ml})$, $11 \%(112 \mathrm{mg} / \mathrm{ml})$, or $20 \%(200 \mathrm{mg} / \mathrm{ml})$ solutions. The $\mathrm{pH}$ of all solutions, including saline, was 5.0. A total volume of $0.5 \mu \mathrm{l}$, resulting in lidocaine doses of $10,30,56$, and $100 \mu \mathrm{g}$, respectively, was infused bilaterally into the rBLA or cBLA at a rate of $0.5 \mu \mathrm{l} / 1.2 \mathrm{~min}$. A piece of PE-20 tubing was used to connect a $5 \mu$ l Hamilton syringe mounted on the syringe pump to a 28 gauge stainless steel infusion cannula that extended $1 \mathrm{~mm}$ beyond the tip of the guide cannula. The infusion cannula was left in place for $1 \mathrm{~min}$ after the infusion. Sterile $0.9 \%$ saline was used as the control vehicle for lidocaine infusions.

\section{Surgery and histology}

Rats were anesthetized with intraperitoneal injections of $90 \mathrm{mg} / \mathrm{kg}$ ketamine plus $10 \mathrm{mg} / \mathrm{kg}$ xylazine. A catheter made from silicon tubing (inner diameter, 0.020 inches; outer diameter, 0.037 inches) was implanted into the right jugular vein as previously described (Kantak et al., 2000). After this procedure, bilateral 22 gauge stainless steel guide cannulas (Plastics One, Roanoke, VA) were stereotaxically implanted according to the atlas of Swanson (1992) $1 \mathrm{~mm}$ above either the rBLA [anteroposterior (AP) $-2.0 \mathrm{~mm}$, lateral (L) $\pm 4.5 \mathrm{~mm}$, dorsoventral (DV) $-7.6 \mathrm{~mm}$ ] or the cBLA (AP $-3.7 \mathrm{~mm}, \mathrm{~L} \pm 5.0 \mathrm{~mm}, \mathrm{DV}-8.2 \mathrm{~mm}$ ) as previously described (Kantak et al., 2001). During this phase of surgery, a $0.1 \mathrm{ml}$ solution containing $1.0 \mathrm{mg}$ methohexital sodium (Brevital; Eli Lilly and Co., Indianapolis, IN) was inf used intraventricularly as needed to maintain anesthesia. Wounds were treated daily until healed with nitrof urazone powder. The catheters were maintained by flushing them daily (Sunday through Saturday) with $0.1 \mathrm{ml}$ of a $0.9 \%$ saline solution containing 0.3 IU of heparin (LymphoMed, Inc., Rosemont, IL), $6.7 \mathrm{mg}$ timentin (SmithKline Beecham Pharmaceuticals, Philadelphia, PA), and 667 IU of streptokinase (Behringwerke AG, Marburg/Lahn, Germany). Catheters were checked for leaks during routine daily flushing. In addition, catheters were checked weekly, or as needed, for function by infusing $0.1 \mathrm{ml}$ of the Brevital solution and noting the presence or absence of sedation. A new catheter was implanted into the left jugular vein to replace a leaky or nonfunctional catheter. If an additional replacement catheter was necessary, it was implanted into the right femoral vein. Rats were allowed to recover from surgery for 1 week before initiation of the study.

After completion of the experiments, rats were given an overdose of sodium pentobarbital and then intracardially perfused with saline and a $10 \%$ formalin solution. Brains were extracted and stored in $10 \%$ formalin. Twenty-four hours before sectioning, brains were transferred to a $20 \%$ glycerin solution stored at $4^{\circ} \mathrm{C}$. Coronal sections $(50 \mu \mathrm{m})$ were cut and stained with thionin to verify placements of cannulas.

\section{Self-administration training}

Training baseline. Before surgery, rats were trained to press a lever under a fixed-ratio 1 (FR1) schedule of food pellet delivery. After food training was complete (rats rapidly pressed the lever for 50 pellets), right jugular vein catheters and guide cannulas were implanted. One week later, $2 \mathrm{hr}$ cocaine self-administration sessions began. Rats were trained to selfadminister $1.0 \mathrm{mg} / \mathrm{kg}$ cocaine under a FI $5 \mathrm{~min}$ (FR5:S) second-order schedule of drug delivery, as previously described (Kantak et al., 2000). Under the FI 5 min (FR5:S) schedule, every fifth lever press (FR5) on the active lever during the $5 \mathrm{~min}$ fixed interval (FI $5 \mathrm{~min}$ ) resulted in the delivery of a $2 \mathrm{sec}$ brief stimulus light located above the active lever. Responses on the inactive lever had no scheduled consequences. For half the rats, the left lever was designated as the active lever and the right lever as the inactive lever. The order was reversed for the remaining rats. Cocaine delivery was made contingent on the completion of an FR5 on the active lever after the $5 \mathrm{~min}$ FI had elapsed. The stimulus light (conditioned stimulus) located above the active lever remained illuminated for the duration of the infusion as well as for a $20 \mathrm{sec}$ time out (TO) period that followed each infusion. The TO period was signaled by the offset of the house light. A maximum of 22 infusions could be earned during a $2 \mathrm{hr}$ session under this second-order schedule. In addition, a 70 $\mathrm{db}$ sound stimulus (discriminative stimulus), either an intermittent $7 \mathrm{kHz}$ tone or continuous white noise (counterbalanced across rats), was presented for the duration of the baseline training sessions. Baseline training sessions, conducted Monday through Friday during the light phase, continued until cocaine intake was stable (number of cocaine infusions did not deviate by $>20 \%$ ) and the number of responses on the inactive lever was no $>25$ over a 5 d period.

Discrimination baseline. After baseline training, the discrimination training method of Weiss et al. (2000) was adapted for use with the second-order schedule of drug delivery to provide self-administration training with unique drug-associated $\left(\mathrm{S}^{+}\right)$and saline-associated $\left(\mathrm{S}^{-}\right)$ sound and light cues. This aspect of training was included because it engenders reliable reinstatement of cue-induced drug-seeking behavior over repeated test sessions without any additional access to cocaine (Weiss et al., 2000). For each day of the $10 \mathrm{~d}$ discrimination-training phase, rats were given two $1 \mathrm{hr}$ self-administration sessions, separated by $1 \mathrm{hr}$. For half the rats, cocaine was available for intravenous delivery during the first session of the day followed by intravenous saline availability during the second session of the day. The order was reversed for the remaining rats. During sessions when cocaine was available, the same intermittent tone or white noise that was presented during the baseline training sessions was presented throughout the $1 \mathrm{hr}$ drug sessions. The light cue was presented during the brief stimulus, drug delivery, and 20 sec TO periods. When saline was available, the opposite sound stimulus as used during the $1 \mathrm{hr}$ drug sessions was presented throughout the $1 \mathrm{hr}$ saline sessions. To provide a unique visual cue during saline sessions, the 
stimulus light located above the active lever flashed during the brief stimulus, saline delivery, and $20 \mathrm{sec}$ TO periods.

Extinction baseline and abstinence. Rats exhibit transient cocaine withdrawal reactions for several days after its termination (Mutschler and Miczek, 1998). Extinction training has been shown to accelerate recovery from cocaine withdrawal (Schmidt et al., 2001). Therefore, to selectively examine amygdaloid control of cue- and drug prime plus cue-induced reinstatement of drug-seeking behavior (see below) without the response-reinstating effects of cocaine withdrawal (Tran-Nguyen et al., 1998), rats first underwent training to extinguish lever responding followed by 1 week of abstinence. During $1 \mathrm{hr}$ extinction sessions, $\mathrm{S}^{+}$and $\mathrm{S}^{-}$sound and light cues were omitted, as was the delivery of cocaine or saline upon making responses on the previously active lever. Extinction training continued until rats made fewer than seven responses on the active lever for three consecutive sessions ( $\sim 15$ sessions). To further disassociate rats from cues associated with the cocaine selfadministration environment, a 1 week abstinence phase was initiated by sequestering the rats in their home cages during this time. Experimental manipulations began after the abstinence phase was complete.

\section{Cue-induced reinstatement of drug-seeking behavior}

Using the training procedures described above, pilot work demonstrated that cocaine-associated cues alone were capable of reliably reinstating drug-seeking behavior for up to six test sessions spaced $3 \mathrm{~d}$ apart (unpublished findings). Therefore, cue-induced reinstatement test sessions were conducted every third day. Rats remained in their home cages on intervening days. To test for the discriminative control over secondorder responding by the stimulus cues, the $\mathrm{S}^{-}$sound cue was presented throughout the first $1 \mathrm{hr}$ test session. The $\mathrm{S}^{-}$light cue was presented for $2 \mathrm{sec}$ after the completion of each FR5 during the $5 \mathrm{~min}$ FI. On completion of each FR5 after the 5 min FI had elapsed, the $\mathrm{S}^{-}$light cue was presented for a period of time equivalent to the duration of an infusion and for the $20 \mathrm{sec}$ TO period. No intravenous infusions (either cocaine or saline) were delivered. To determine if lidocaine inactivation of different sites within the basolateral amygdala could disrupt reinstatement of drug-seeking behavior maintained by cocaine-associated cues, either $10 \mu \mathrm{g} / 0.5 \mu \mathrm{l}$ lidocaine or $0.5 \mu \mathrm{l} 0.9 \%$ saline were bilaterally infused into the rBLA $(n=8)$ or $\operatorname{cBLA}(n=7) 5 \mathrm{~min}$ before the second and third test sessions. Order of infusions was counterbalanced across rats. Because cocaine was not used during the cue-induced reinstatement tests, only the $10 \mu \mathrm{g}$ dose of lidocaine was evaluated. Previous work demonstrated that bilateral infusion of $10 \mu \mathrm{g}$ lidocaine was sufficient to fully disrupt cue-based cognitive task performance (Kantak et al., 2001), thus making the use of higher doses in the cue-induced reinstatement tests redundant. During these test sessions, the $\mathrm{S}^{+}$sound cue was presented throughout each $1 \mathrm{hr}$ session. The $\mathrm{S}^{+}$light cue was presented as above for the $\mathrm{S}^{-}$light cue. Again, no intravenous infusions were delivered.

\section{Cocaine prime plus cue-induced reinstatement of drug-seeking behavior}

After completion of the cue-induced reinstatement procedure, test sessions were conducted in the same rats to examine cocaine prime plus cue-induced reinstatement of drug-seeking behavior. For this test, an intraperitoneal priming injection of $20 \mathrm{mg} / \mathrm{kg}$ cocaine, which produces maximal reinstatement of drug-seeking behavior in Wistar strain rats (Mantsch and Goeders, 1999) was administered $30 \mathrm{~min}$ before the session. The 30 min postinjection interval was used to minimize the response-decreasing effects of a noncontingent injection of cocaine administered before self-administration sessions (Markou et al., 1999). Rats were bilaterally infused with saline, $10,30,56$, or $100 \mu \mathrm{g}$ lidocaine in a counterbalanced order $5 \mathrm{~min}$ before the session. Each test was conducted $3 \mathrm{~d}$ apart. Higher doses of lidocaine were evaluated in this study because preliminary tests revealed that $10 \mu \mathrm{g}$ of lidocaine was not sufficient to alter responding when a cocaine priming injection preceded the lidocaine infusion by $25 \mathrm{~min}$. As above, the $\mathrm{S}^{+}$sound cue was presented throughout each $1 \mathrm{hr}$ session. The $\mathrm{S}^{+}$light cue was presented for $2 \mathrm{sec}$ after the completion of each FR5 during the $5 \mathrm{~min}$ FI. After the completion of each FR5 after the 5 min FI had elapsed, the $\mathrm{S}^{+}$light cue was presented for a period of time equivalent to the duration of an infusion and for the $20 \mathrm{sec}$ TO period. No intravenous infusions were delivered.

\section{Drug-seeking and drug-taking behavior under cocaine maintenance conditions}

To directly examine the effects of lidocaine inactivation of the rBLA and cBLA on drug-taking behavior during repeated cocaine availability, three rats ( $n=2 \mathrm{rBLA}$ and $n=1 \mathrm{cBLA}$ ) used in the above reinstatement experiments and five experimentally naive rats $(n=2 \operatorname{rBLA}$ and $n=3$ cBLA) were examined under cocaine maintenance conditions. After establishing or reestablishing stable cocaine baselines and discrimination sessions were underway, rats from each group $(n=4)$ were bilaterally inf used with saline, $10,30,56$, or $100 \mu \mathrm{g}$ lidocaine in a counterbalanced order $5 \mathrm{~min}$ before a cocaine self-administration test session. Each $1 \mathrm{hr}$ test session was conducted $3 \mathrm{~d}$ apart, with discrimination sessions on intervening days. The $\mathrm{S}^{+}$sound cue was presented throughout each test session, and the $\mathrm{S}^{+}$light cue was presented during the brief stimulus, drug delivery, and $20 \mathrm{sec}$ TO periods.

\section{Specificity of the behavioral effects of lidocaine}

To determine if the reductions in drug-seeking behavior after lidocaine treatment were attributable to a nonspecific disruption in lever responding, a small group $(n=3)$ of the same rats as used in the reinstatement experiments (two from the cBLA group; one from the rBLA group) were examined for the effects of lidocaine on responding maintained by a FR5 schedule of food pellet delivery. No sound or light stimuli were used. After baseline responding was stable, rats were infused with $100 \mu \mathrm{g}$ of lidocaine $5 \mathrm{~min}$ before a single $1 \mathrm{hr}$ test session.

\section{Data analysis}

For the self-administration studies, three dependent measures were calculated: (1) the total number of active lever responses emitted during the session (drug-seeking behavior), (2) the total number of inactive lever responses emitted during the session (non-specific responding), and (3) the total number of infusions earned during maintenance tests (drug-taking behavior) or the total number of infusion-paired light deliveries earned during reinstatement tests. Data from individual animals were averaged over the last three cocaine, saline, and extinction sessions and used to portray baseline performance. A series of singlefactor (condition or dose) ANOVAs with repeated measures was performed for each brain site, and the Dunnett's $t$ test was used for all post hoc comparisons. Paired $t$ tests for dependent samples were used to compare the number of infusions earned during baseline sessions. For the food study, the number of active and inactive lever responses as well as pellets earned during baseline (averaged over the last three sessions) and after bilateral infusions of $100 \mu \mathrm{g}$ lidocaine were calculated. The two conditions were compared by paired $t$ tests for dependent samples.

\section{RESULTS}

\section{Functional spread of lidocaine and histology}

The volume of lidocaine required to inactivate $>90 \%$ of neurons within a particular radius from the infusion site is governed by the spherical volume equation, $V=4 / 3 \pi \mathrm{r}^{3}$ (Tehovnik and Sommer, 1997). Collectively, several studies (Sandkühler and Gebhart, 1984; Sandkühler et al., 1987; Tehovnik and Sommer, 1997; Malpeli, 1999; Martin and Ghez, 1999) have shown that as lidocaine infusion volume increases (from 0.1 to $10 \mu \mathrm{l}$ ), the radius of the functional spread increases in a fairly linear manner (from 0.25 to $2.0 \mathrm{~mm}$ ). Furthermore, the spread of lidocaine appears to be less dependent on concentration, but higher concentrations may prolong the time course of inactivation. Generally, the effects of lidocaine completely disappear after 30-90 min in sites having a functional spread zone with radii of 0.1-2.0 $\mathrm{mm}$ (Lomber, 1999).

Based on the spherical volume equation, the functional spread of $0.5 \mu \mathrm{l}$ lidocaine at each concentration is estimated to have been $0.49 \mathrm{~mm}$ from the infusion site in the present study. Consistent with this calculation, conduction analyses in the medulla or spinal cord show that $0.5 \mu \mathrm{l}$ of lidocaine infused at fourfold different concentrations block an area with a radius of $0.45-0.50 \mathrm{~mm}$ from the infusion site (Sandkühler and Gebhart, 1984; Sandkühler et al., 1987). Using glucose uptake as a marker, it has also been 
Rostral Basolateral Amygdala

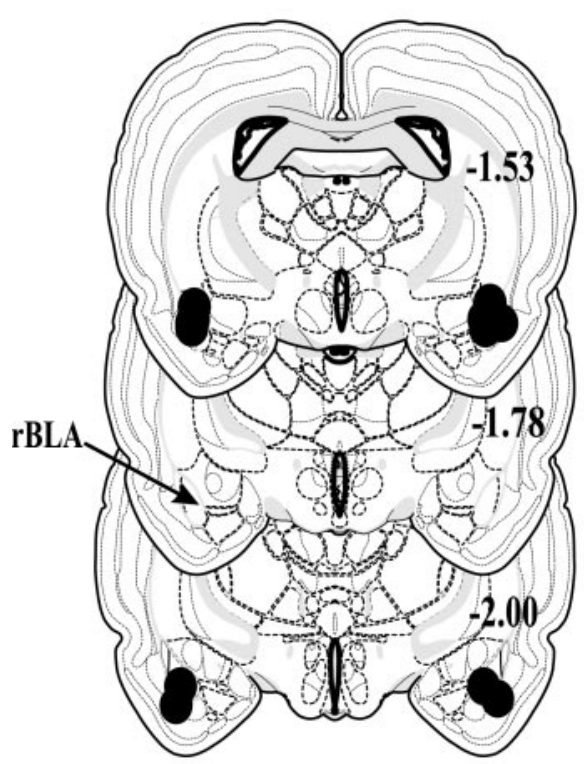

Caudal Basolateral Amygdala

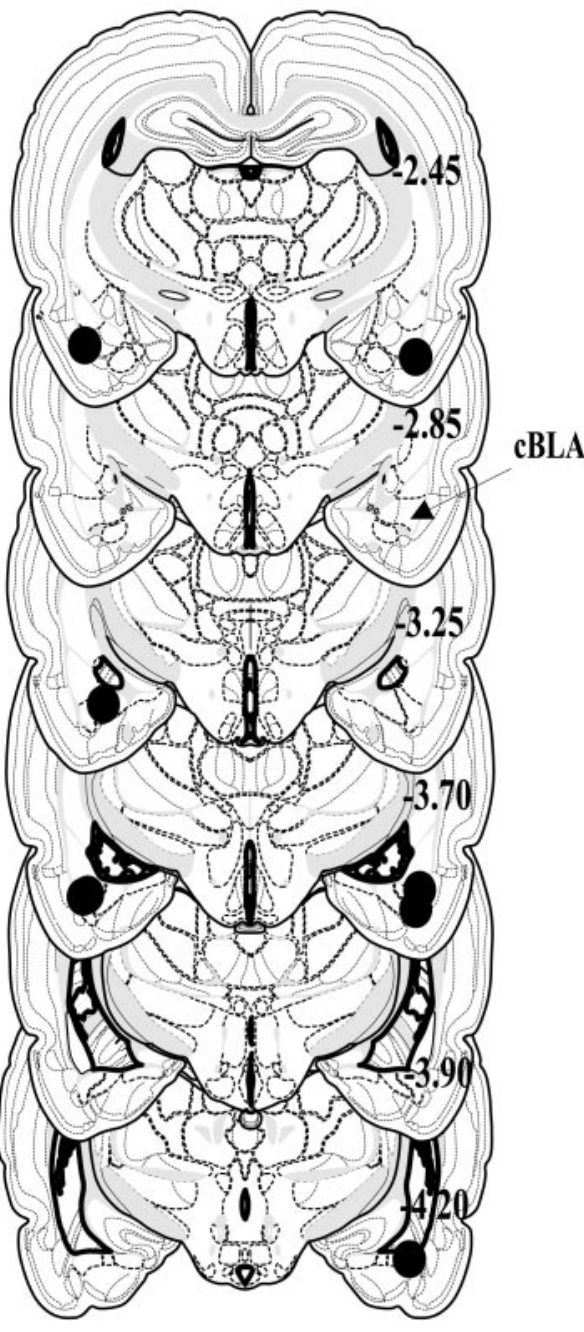

Figure 1. Schematic representing coronal sections of the rat brain and cannula placements within the rostral (left) and caudal (right) regions of the basolateral amygdala. Circles indicate the location and diffusion of lidocaine and are drawn to scale. All drawings are based on the atlas of Swanson (1992) with the anterior-posterior reference measured from bregma. Each placement is shown at its midpoint along the anterior-posterior plane. noted that the effects of focal inactivation by lidocaine extend beyond the region of drug spread (Martin, 1991; Martin and Ghez, 1999). The hypometabolism extending beyond the central core of inactivation is thought to be attributable to reduced synaptic activity of neurons receiving projections directly from the inactivated region rather than additional spread of lidocaine. Based on these considerations, we assumed a functional spread zone with a radius of $0.49 \mathrm{~mm}$ in the analysis and interpretation of the results described below.

Histological verification of bilateral placements was confirmed for 8 of 10 rats with cannulas aimed at the rBLA (Fig. 1, left). For every animal, cannulas were within $0.5 \mathrm{~mm}$ of the intended placement in the anterior-posterior position and within the rBLA anatomical range (Swanson, 1992). The only brain structure within the indicated functional spread zone that was common to all animals was the rBLA. Diffusion to the endopiriform nucleus, stria terminalis, basomedial amygdala nucleus, and lateral amygdala nucleus was apparent in some rats.

Histological verification of bilateral placements was confirmed for 8 of 10 rats with cannulas aimed at the cBLA (Fig. 1, right). All cannulas were within $1.3 \mathrm{~mm}$ of the intended placement in the anterior-posterior position and within the cBLA anatomical range (Swanson, 1992). The only brain structure within the indicated functional spread zone that was common to all rats was the
cBLA. Diffusion to the endopiriform nucleus, lateral amygdala nucleus, and basomedial amygdala nucleus was apparent in some rats. In addition, minor unilateral diffusion into the lateral ventricles may have occurred in two of the eight rats (one used only in the maintenance experiment and one used only in the reinstatement experiments).

The above analyses indicate that there was a sufficient separation of placements from the rostral versus caudal BLA to delineate the two discrete regions of interest. Animals with cannulas that were not bilaterally positioned in the intended site were omitted from further analyses. Furthermore, for all rats included in the analyses, cannulas placements verify that lidocaine inactivation predominantly encompassed the region of interest. A representative photomicrograph of the extent of damage made by 22 gauge guide cannulas and a tract made by a 28 gauge infusion cannula is shown in Figure 2 for an rBLA-implanted rat.

\section{Cue-induced reinstatement of drug-seeking behavior Rostral BLA}

As shown in Figure 3 (top), cocaine maintained high rates of active lever responding that were clearly distinguished from saline performance during baseline discrimination sessions and from extinction performance during extinction sessions $\left(F_{(2,10)}=\right.$ 
Figure 2. Representative photomicrograph of the extent of damage made by 22 gauge guide cannulas. In this example, the tips of the guide cannulas were bilaterally positioned $1 \mathrm{~mm}$ above the rostral basolateral amygdala at a level $2.0 \mathrm{~mm}$ posterior to bregma. A tract made by a 28 gauge infusion cannula is visible in the left hemisphere.

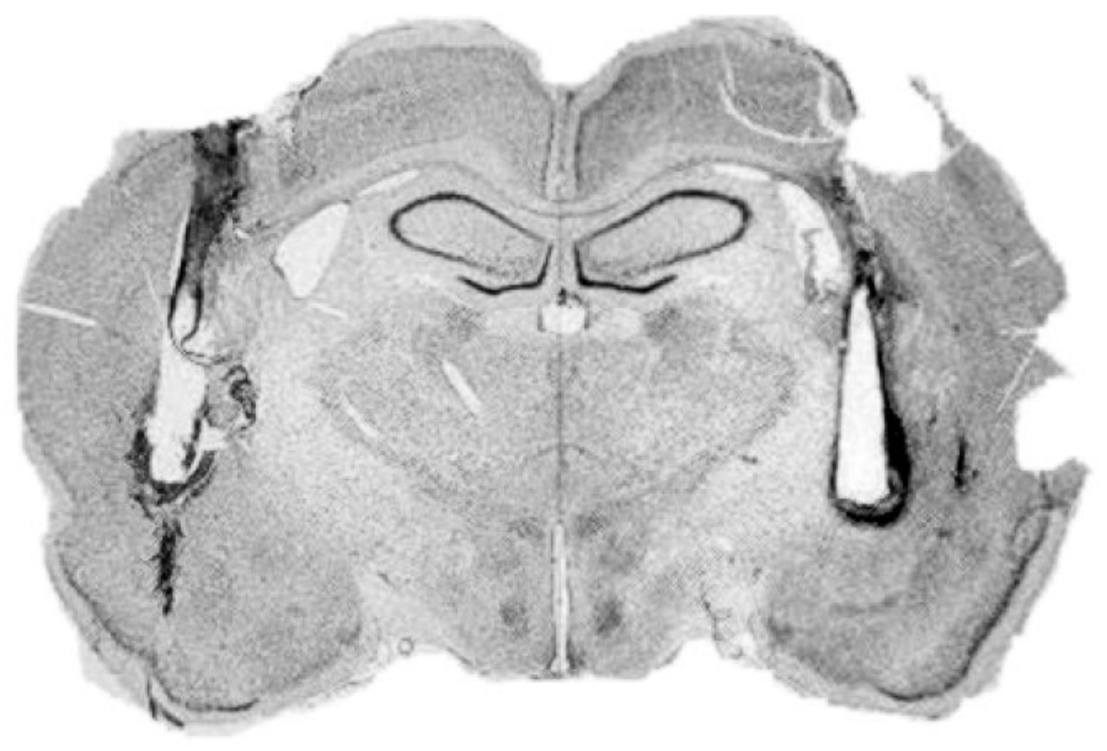

$42 ; p \leq 0.001$ ). Responses on the inactive lever (Fig. 3, bottom) averaged $\leq 5$ during baseline as well as during extinction and both reinstatement tests, with no significant differences among test conditions ( $p \leq 0.21-0.50)$. As expected, the number of inf usions earned during cocaine baseline sessions (Fig. 4) was significantly greater than the number earned during saline baseline sessions $\left(t_{(5)}=22 ; p \leq 0.001\right)$.

After extinction training, $\mathrm{S}^{+}$cues associated with cocaine availability reinstated drug-seeking behavior on the active lever to $68 \%$ of the cocaine baseline, whereas $\mathrm{S}^{-}$cues associated with saline availability resulted in saline baseline-like levels of responding (Fig. 3, top). This difference in active lever responses under $\mathrm{S}^{+}$and $\mathrm{S}^{-}$control conditions was significant $\left(F_{(2,10)}=12\right.$; $p \leq 0.002)$. The difference under $\mathrm{S}^{+}$and $\mathrm{S}^{-}$control conditions was significant for the number of inf usion-paired light deliveries earned as well $\left(F_{(2,10)}=27 ; p \leq 0.001\right)$ (Fig. 4, top). Compared with the $\mathrm{S}^{+} 0 \mu \mathrm{g}$ lidocaine control, bilateral infusions of $10 \mu \mathrm{g}$ lidocaine into the rBLA completely blocked the reinstatement of drug-seeking behavior produced by the conditioned $\mathrm{S}^{+}$cocaine cues and subsequently greatly reduced the number of infusionpaired light deliveries earned. The degree to which the $\mathrm{S}^{+}$drug cues reinstated drug-seeking behavior and the degree to which this behavior was blocked after lidocaine inactivation was not related to baseline levels of drug-seeking behavior $(r=0.71, p \leq$ 0.12 and $r=-0.21, p \leq 0.82$, respectively)

\section{Caudal BLA}

The baseline performance profile in rats with cannulas aimed at the cBLA was similar to the profile observed in rats with cannulas aimed at the rBLA. Drug-seeking behavior on the active lever (Fig. 5, top) $\left(F_{(2,8)}=14 ; p \leq 0.002\right)$ and the number of infusions earned (Fig. 6) $\left(t_{(4)}=15 ; p \leq 0.001\right)$ were significantly greater during cocaine availability than during saline availability or extinction. The greater average number of responses observed during cocaine baseline sessions in the cBLA group compared to the rBLA group was predominantly attributable to an extremely high level of active lever responding in one subject from the cBLA group. Inactive lever responses (Fig. 5, bottom) averaged $\leq 6$ across baseline, extinction, and both reinstatement tests, and there were no significant differences among test conditions ( $p \leq$ $0.14-0.82)$.
After extinction training, $\mathrm{S}^{+}$cues associated with cocaine availability reinstated drug-seeking behavior on the active lever to $20 \%$ of the cocaine baseline in this group of rats, whereas $\mathrm{S}^{-}$cues associated with saline availability resulted in saline baseline-like levels of responding (Fig. 5, top). This difference in active lever responses under $\mathrm{S}^{+}$and $\mathrm{S}^{-}$control conditions was significant $\left(F_{(2,8)}=14 ; p \leq 0.002\right)$. The difference under $\mathrm{S}^{+}$and $\mathrm{S}^{-}$control conditions was significant for the number of infusion-paired light deliveries earned as well $\left(F_{(2,8)}=6.7 ; p \leq 0.02\right)$ (Fig. 6). Compared with the $\mathrm{S}^{+} 0 \mu \mathrm{g}$ lidocaine control, bilateral infusions of 10 $\mu \mathrm{g}$ lidocaine into the cBLA did not significantly alter the reinstatement of drug-seeking behavior or number of infusion-paired light deliveries earned after exposure to the conditioned $\mathrm{S}^{+}$ cocaine cues. Even after $t$ test analysis of reinstated drug-seeking behavior induced by the $\mathrm{S}^{+}$drug cues, the effects of lidocaine inactivation were not significantly different from the effects observed after saline infusion $(p \leq 0.11)$. The degree to which the $\mathrm{S}^{+}$drug cues reinstated drug-seeking behavior after saline or lidocaine inactivation was not related to baseline levels of drugseeking behavior $(r=0.68, p \leq 0.20$ and $r=0.60, p \leq 0.28$, respectively).

\section{Cocaine prime plus cue-induced reinstatement of drug-seeking behavior}

Rostral BLA

After an intraperitoneal priming injection of $20 \mathrm{mg} / \mathrm{kg}$ cocaine, the conditioned $\mathrm{S}^{+}$cocaine cues reinstated drug-seeking behavior on the active lever to $145 \%$ of the cocaine baseline (Fig. 3, top). Bilateral lidocaine infusions into the rBLA dosedependently attenuated the reinstatement of drug-seeking behavior, with a significant reduction after $100 \mu \mathrm{g}\left(F_{(4,16)}=3.3 ; p \leq\right.$ 0.04). Drug-seeking behavior was reduced without a corresponding change in the number of infusion-paired light deliveries earned (Fig. 4) $(p \leq 0.27)$.

\section{Caudal BLA}

A cocaine priming injection in combination with the conditioned $\mathrm{S}^{+}$cocaine cues reinstated drug-seeking behavior on the active lever to $35 \%$ of the cocaine baseline in this group of rats (Fig. 5, top). Bilateral lidocaine infusions into the cBLA dose- 


\section{Rostral Basolateral Amygdala}

\section{Drug-Seeking Behavior}
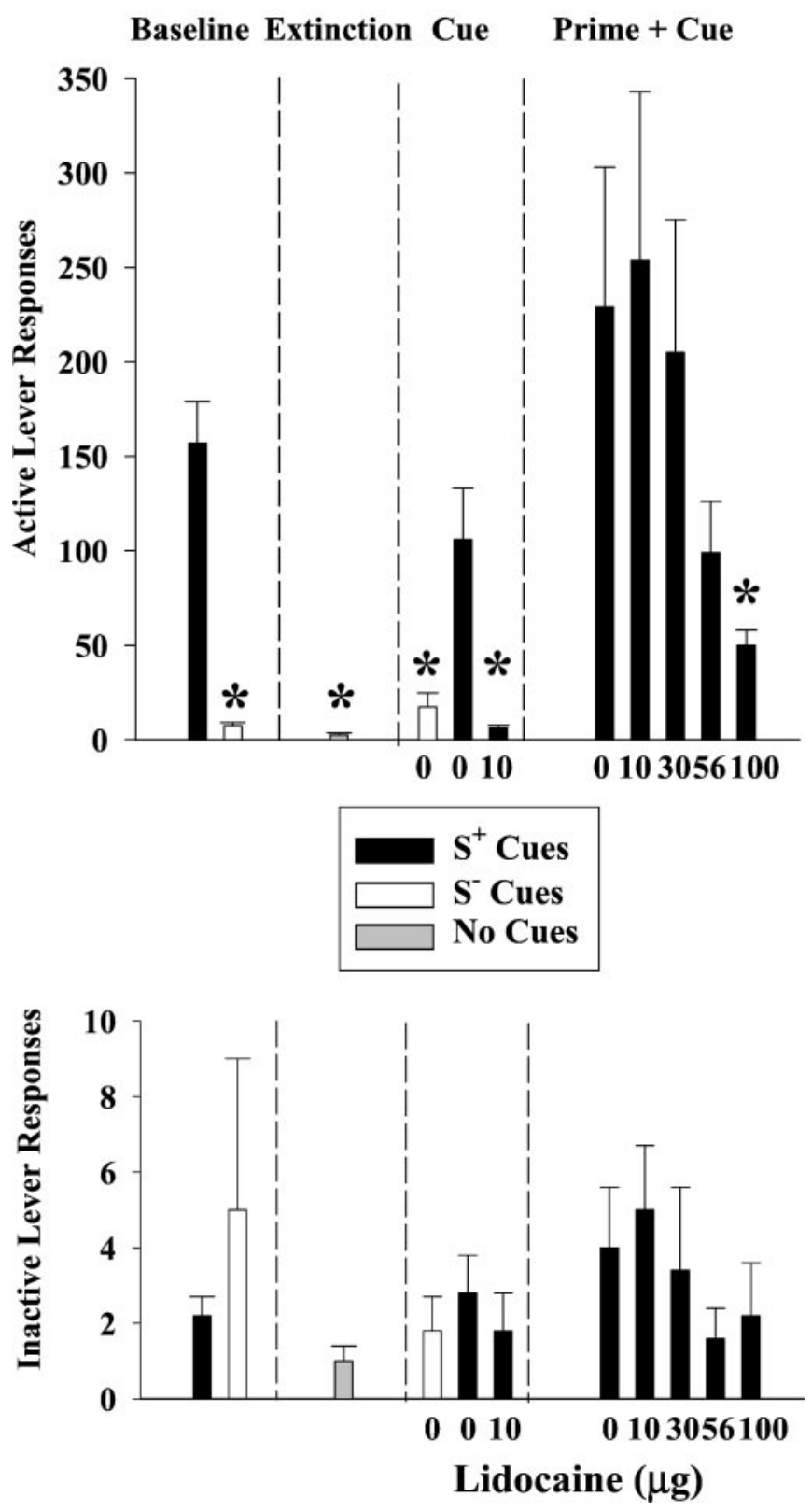

Figure 3. Drug-seeking behavior after lidocaine inactivation of the rostral basolateral amygdala $(n=6)$. Responses on the active lever are shown on the top, and responses on the inactive lever are shown on the bottom for each phase of the experiment: discrimination baseline, extinction, drug cue-induced reinstatement, and drug prime plus drug cue-induced reinstatement conditions. Values are the mean \pm SEM. $\mathrm{S}^{+}$refers to the drug-associated cues, and $\mathrm{S}^{-}$refers to the saline-associated cues. ${ }^{*}$ Significantly different $(p \leq 0.05)$ from the appropriate control value. See Results for statistical details.

dependently attenuated the reinstatement of drug-seeking behavior, with significant reductions after 56 and $100 \mu \mathrm{g}\left(F_{(4,12)}=3.3\right.$; $p \leq 0.05)$. Drug-seeking behavior was reduced without a corresponding change in the number of infusion-paired light deliveries earned (Fig. 6) $(p \leq 0.27)$.

\section{Rostral Basolateral Amygdala}

\section{Infusions or Infusion-Paired Light Deliveries}

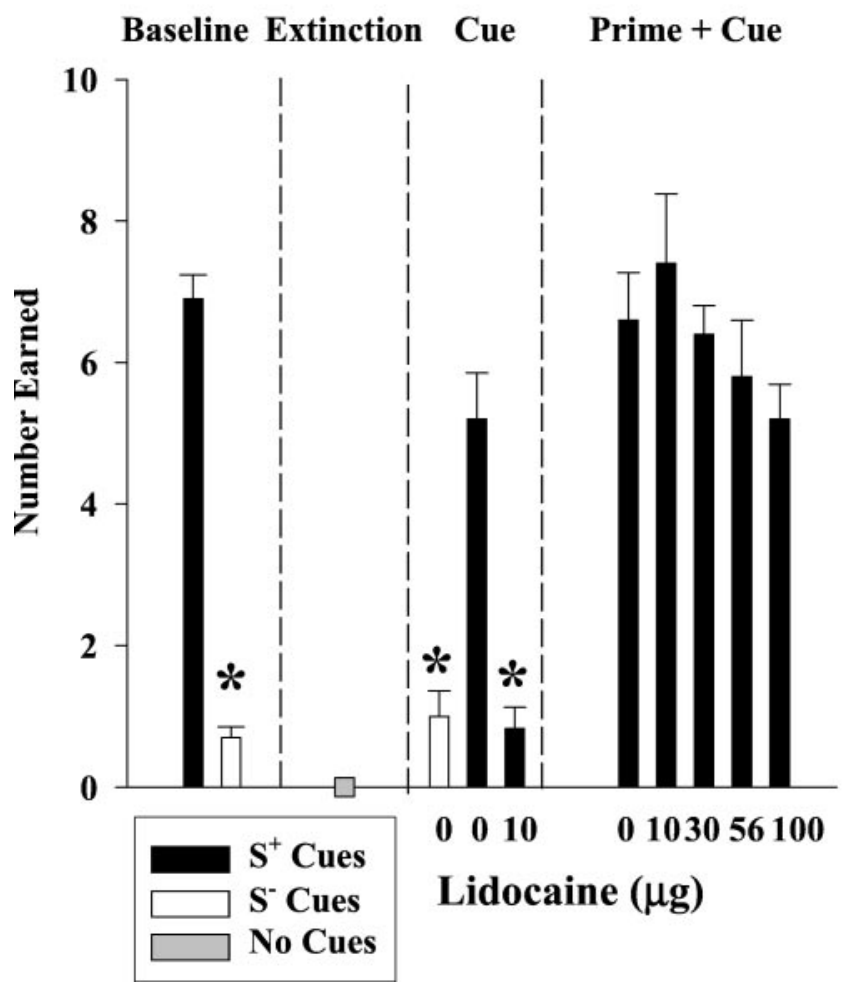

Figure 4. Number of infusions earned or number of inf usion-paired light deliveries earned after lidocaine inactivation of the rostral basolateral amygdala for each phase of the experiment: discrimination baseline, extinction, cue-induced reinstatement, and prime plus cue-induced reinstatement conditions $(n=6)$. Values are the mean \pm SEM. $\mathrm{S}^{+}$refers to the drug-associated cues, and $\mathrm{S}^{-}$refers to the saline-associated cues. *Significantly different $(p \leq 0.05)$ from the appropriate control value. See Results for statistical details.

To ensure that the differential effects of lidocaine dose during the cue versus prime plus cue tests was not attributable to differences in the time course of inactivation, two-factor repeated measures ANOVAs were conducted to assess changes in responding throughout the $1 \mathrm{hr}$ test sessions (Table 1). These analyses indicate that when a dose of lidocaine was effective in reducing the reinstatement of drug-seeking behavior, it did so throughout the $1 \mathrm{hr}$ test session. This occurred whether the dose was $10 \mu \mathrm{g}$ (rBLA cue test) or 56-100 $\mu \mathrm{g}$ (rBLA and cBLA prime plus cue tests). In the prime plus cue tests, doses of lidocaine $<56 \mu \mathrm{g}$ were primarily ineffective throughout the $15 \mathrm{~min}$ intervals. Thus, there was no tendency for lower doses of lidocaine to be effective only in the early segments of the test sessions, which could potentially mask significant effects when data are collapsed over the entire $1 \mathrm{hr}$.

Drug-seeking and drug-taking behavior under cocaine maintenance conditions

\section{Rostral BLA}

During cocaine maintenance test sessions, bilateral lidocaine inactivation of the rBLA with doses up to $100 \mu \mathrm{g}$ did not modify drug-seeking $(p \leq 0.59)$ or drug-taking $(p \leq 0.62)$ behavior (Fig. 


\section{Caudal Basolateral Amygdala}

\section{Drug-Seeking Behavior}
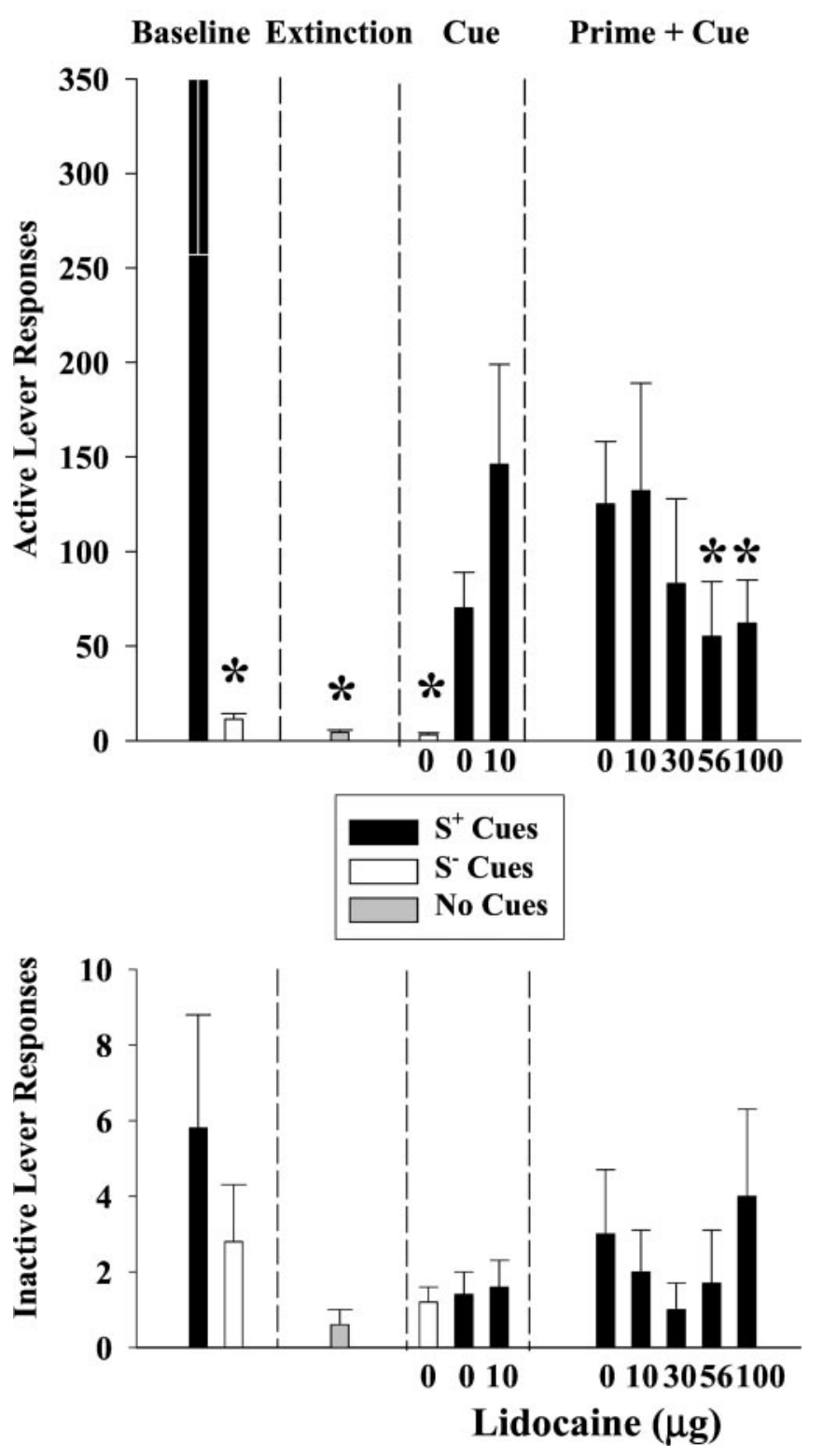

Figure 5. Drug-seeking behavior after lidocaine inactivation of the caudal basolateral amygdala $(n=5)$. Responses on the active lever are shown on the top, and responses on the inactive lever are shown on the bottom for each phase of the experiment: discrimination baseline, extinction, drug cue-induced reinstatement, and drug prime plus drug cue-induced reinstatement conditions. Values are the mean \pm SEM. $\mathrm{S}^{+}$refers to the drug-associated cues, and $\mathrm{S}^{-}$refers to the saline-associated cues. *Significantly different $(p \leq 0.05)$ from the appropriate control value. See Results for statistical details.

7 , top and bottom left). Inactive lever responses averaged $\leq 3$, and there were no significant differences across lidocaine doses $(p \leq$ $0.99)$.

\section{Caudal BLA}

In contrast to the rBLA, bilateral lidocaine inactivation of the cBLA during cocaine maintenance test sessions resulted in re-

\section{Caudal Basolateral Amygdala}

\section{Infusions or Infusion-Paired Light Deliveries}

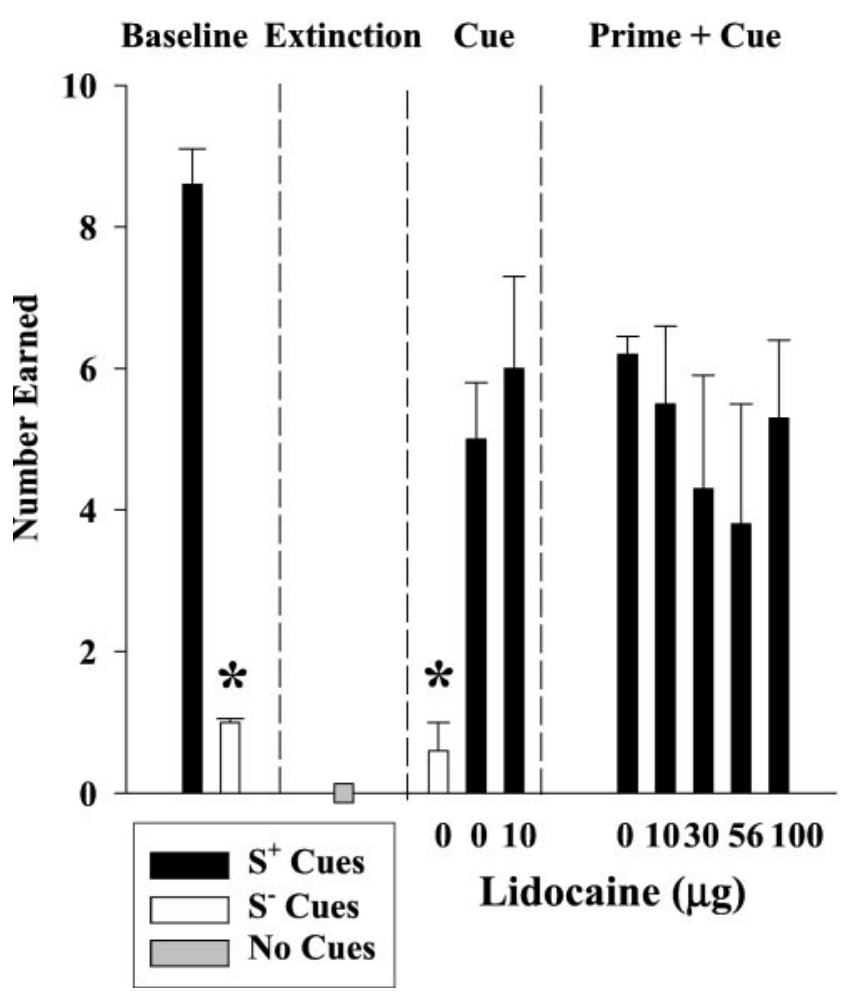

Figure 6. Number of inf usions earned or number of inf usion-paired light deliveries earned after lidocaine inactivation of the caudal basolateral amygdala for each phase of the experiment: discrimination baseline, extinction, drug cue-induced reinstatement, and drug prime + drug cue-induced reinstatement conditions $(n=5)$. Values are the mean \pm SEM. $\mathrm{S}^{+}$refers to the drug-associated cues, and $\mathrm{S}^{-}$refers to the salineassociated cues. *Significantly different $(p \leq 0.05)$ from the appropriate control value. See Results for statistical details.

ductions in drug-seeking behavior (Fig. 7, top right). Significant reductions were observed after 56 and $100 \mu \mathrm{g}\left(F_{(3,9)}=6.57 ; p \leq\right.$ $0.01)$. Drug-seeking behavior was reduced without a corresponding change in drug-taking behavior (Fig. 7, bottom right) ( $p \leq$ $0.15)$ or inactive lever responses $(p \leq 0.38)$, which averaged $\leq 8$ across lidocaine doses.

\section{Specificity of the behavioral effects of lidocaine}

To determine if the reductions in responding after infusion with $100 \mu \mathrm{g}$ lidocaine were attributable to nonspecific disruption of responding in general, rats were trained on a FR5 schedule of food pellet delivery for 3 weeks and then bilaterally inf used with $100 \mu \mathrm{g}$ of lidocaine in a single test session (Fig. 8). There were no significant differences between baseline performance and performance after $100 \mu \mathrm{g}$ of lidocaine in active lever responses $(p \leq$ $0.40)$, inactive lever responses $(p \leq 0.25)$, or pellets earned ( $p \leq$ $0.41)$. These findings indicate that bilateral infusion of $100 \mu \mathrm{g}$ lidocaine into either region of the BLA does not produce nonspecific reductions in responding.

\section{DISCUSSION}

The present study outlines a procedure in rats whereby a high degree of drug-seeking behavior was induced during mainte- 


\begin{tabular}{|c|c|c|c|c|c|c|c|c|}
\hline \multirow{2}{*}{$\frac{\text { rBLA }}{\text { Cues }}$} & \multicolumn{3}{|c|}{ Cue-induced reinstatement ${ }^{a}$} & \multicolumn{5}{|c|}{ Prime + cue-induced reinstatement ${ }^{b}$} \\
\hline & $\mathrm{S}^{-}$ & $\mathrm{S}^{+}$ & $\mathrm{S}^{+}$ & $\mathrm{S}^{+}$ & $\mathrm{S}^{+}$ & $\mathrm{S}^{+}$ & $\mathrm{S}^{+}$ & $\mathrm{S}^{+}$ \\
\hline Condition/dose $(\mu \mathrm{g})$ & 0 & 0 & 10 & 0 & 10 & 30 & 56 & 100 \\
\hline $0-15$ & $20(16)$ & $76(39)$ & $2(1)$ & $44(25)$ & $48(22)$ & $32(25)$ & $15(10)$ & $11(5)$ \\
\hline $15-30$ & $4(4)$ & $20(3)$ & $3(2)$ & $42(25)$ & $51(27)$ & $40(14)$ & $23(12)$ & $9(3)$ \\
\hline $30-45$ & $2(1)$ & $12(4)$ & $.2(.2)$ & $66(22)$ & $57(39)$ & $28(8)$ & $30(5)$ & $15(3)$ \\
\hline $45-60$ & $.4(.4)$ & $22(10)$ & $2(1)$ & $61(25)$ & $30(19)$ & $41(7)$ & $19(9)$ & $18(6)$ \\
\hline cBLA & \multicolumn{3}{|c|}{ Cue-induced reinstatement ${ }^{c}$} & \multicolumn{5}{|c|}{ Prime + cue-induced reinstatement ${ }^{d}$} \\
\hline Cues & $\mathrm{S}^{-}$ & $\mathrm{S}^{+}$ & $\mathrm{S}^{+}$ & $\mathrm{S}^{+}$ & $\mathrm{S}^{+}$ & $\mathrm{S}^{+}$ & $\mathrm{S}^{+}$ & $\mathrm{S}^{+}$ \\
\hline Condition/dose $(\mu \mathrm{g})$ & 0 & 0 & 10 & 0 & 10 & 30 & 56 & 100 \\
\hline $0-15$ & $1(.6)$ & $24(12)$ & $50(24)$ & $19(12)$ & $38(27)$ & $15(9)$ & $21(17)$ & $11(6)$ \\
\hline $15-30$ & $0(0)$ & $7(3)$ & $37(16)$ & $11(8)$ & $31(17)$ & $30(19)$ & $12(9)$ & $24(12)$ \\
\hline $30-45$ & $3(3)$ & $15(8)$ & $23(7)$ & $37(9)$ & $33(9)$ & $31(20)$ & $12(6)$ & $11(4)$ \\
\hline $45-60$ & $3(2)$ & $24(9)$ & $37(21)$ & $82(44)$ & $31(19)$ & $7(3)$ & $10(5)$ & $16(4)$ \\
\hline
\end{tabular}

\begin{tabular}{|c|c|c|c|}
\hline \multicolumn{4}{|l|}{ ANOVA analyses: } \\
\hline Condition $^{a}$ & $F_{(2,10)}=6.6 ; p \leq 0.02$ & $\operatorname{Dose}^{b}$ & $F_{(4,20)}=4.7 ; p \leq 0.01$ \\
\hline Time & $F_{(3,15)}=2.2 ; p \leq 0.13$ & Time & $F_{(3,15)}=2.2 ; p \leq 0.13$ \\
\hline Condition $\times$ time & $F_{(6,30)}=2.3 ; p \leq 0.10$ & Dose $\times$ time & $F_{(12,60)}=0.8 ; p \leq 0.63$ \\
\hline Condition $^{c}$ & $F_{(2,8)}=5.9 ; p \leq 0.03$ & $\operatorname{Dose}^{d}$ & $F_{(4,16)}=3.9 ; p \leq 0.02$ \\
\hline Time & $F_{(3,12)}=0.8 ; p \leq 0.52$ & Time & $F_{(3,12)}=0.7 ; p \leq 0.60$ \\
\hline Condition $\times$ time & $F_{(6,24)}=0.6 ; p \leq 0.68$ & Dose $\times$ time & $F_{(12,48)}=2.8 ; p \leq 0.01$ \\
\hline
\end{tabular}

Values are the mean (SEM) responses for successive 15 min periods during 1 hr test sessions.

${ }^{a, b}$ ANOVA analyses in rBLA implanted rats.

${ }^{c, d}$ ANOVA analyses in cBLA implanted rats.

nance testing and reinstated by drug-associated sound and light cues after a period of extinction and abstinence from cocaine self-administration. Factors contributing to robust levels of response reinstatement are use of multiple stimulus cues (See et al., 1999), unique discriminative stimuli to predict drug availability and nonavailability (Weiss et al., 2000), and a second-order schedule of drug delivery (Spealman et al., 1999). As shown here, a second-order schedule of cocaine delivery with discriminative and conditioned drug cues reinstated responding to levels that were fivefold to sixfold higher than levels reported by others using FR schedules of cocaine delivery (Meil and See, 1997; Grim and See, 2000; Weiss et al., 2000). Consistent with nonhuman primate studies, response reinstatement by drug-associated cues was even greater after cocaine priming injections (Spealman et al., 1999). As such, these findings indicate that the presently developed model is robust and reliable for studying neurobiological mechanisms of drug-seeking and drug-taking behavior under both maintenance and reinstatement conditions in rats. The present findings suggest that reinstatement of drug-seeking behavior is dependent on activation of the rBLA under abstinence conditions (no cocaine for 4 weeks). However, the caudal region may function cooperatively with the rostral region to reinstate drugseeking behavior after brief exposure to cocaine (single intraperitoneal cocaine injection). After repeated exposure to cocaine during maintenance sessions (repeated intravenous cocaine infusions), regulation of drug-seeking behavior is dependent on activation of the cBLA.

\section{Dissociable effects of lidocaine inactivation of the rBLA and cBLA}

In the rBLA, a dose of lidocaine $(10 \mu \mathrm{g})$ that fully blocked the expression of conditioned stimulus-reward learning in a condi- tioned cue preference cognitive task (Kantak et al., 2001) also fully blocked the expression of drug-seeking behavior reinstated by cocaine-associated sound and light cues. Previous cocaine self-administration studies, which used either excitotoxic lesions (Meil and See, 1997) or TTX inactivation (Grim and See, 2000), demonstrated that disturbance within an area of the basolateral nucleus that primarily encompassed the rostral region also fully blocked reinstatement of drug-seeking behavior induced by cocaine-associated cues. In the TTX study, responding maintained by repeated availability of cocaine was not altered after infusion of TTX. In agreement with this finding, inactivation of the rBLA with lidocaine (up to $100 \mu \mathrm{g}$ ) also did not alter drugseeking or drug-taking behavior maintained by repeated cocaine availability. In contrast, after a cocaine-priming injection, $100 \mu \mathrm{g}$ of lidocaine infused into the rBLA attenuated the reinstatement of drug-seeking behavior. Despite this reduction, the number of infusion-paired light deliveries earned was not altered.

Although the cBLA is also involved in regulating conditioned stimulus-reward learning (Kantak et al., 2001), a dissociation of the effects of lidocaine inactivation in the rBLA and cBLA on drug-seeking behavior was evident. In the cBLA, $10 \mu \mathrm{g}$ of lidocaine did not alter drug-seeking behavior reinstated by cocaineassociated sound and light cues. Higher doses of lidocaine (56 and $100 \mu \mathrm{g})$, however, did attenuate drug-seeking behavior reinstated by a cocaine priming injection plus cues, without producing an effect on the number of infusion-paired light deliveries earned. Importantly, under cocaine maintenance conditions, lidocaine inactivation of the cBLA attenuated drug-seeking behavior without altering drug-taking behavior. Dissociable effects on drugseeking behavior versus drug intake were also reported by Whitelaw et al. (1996), who examined the effects of excitotoxic 


\section{Maintenance}
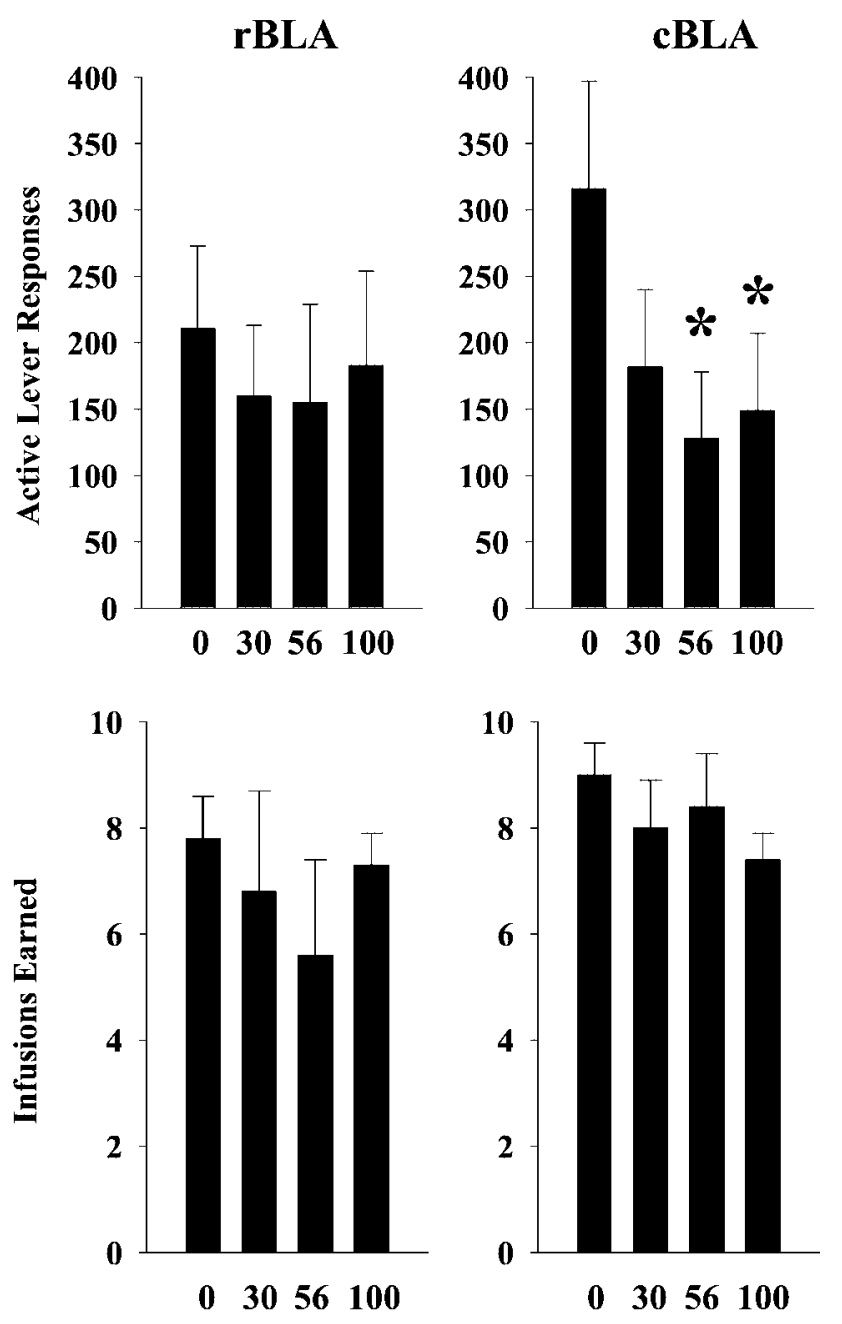

\section{Lidocaine $(\mu \mathrm{g})$}

Figure 7. Drug-seeking (top) and drug-taking (bottom) behavior after lidocaine inactivation of the rostral basolateral amygdala (left $)$ and caudal basolateral amygdala (right) during maintenance testing $(n=4$ for each site). Values are the mean \pm SEM.

damage to the BLA (encompassing both rostral and caudal regions) on acquisition of cocaine self-administration behavior studied under a second order-fixed ratio schedule of drug delivery. Together, these findings suggest that independent neural mechanisms might control drug-seeking and drug-taking behavior.

Although lidocaine infusions into the rBLA and cBLA effectively attenuated the reinstatement of drug-seeking behavior after a cocaine priming injection, a 5- to 10 -fold higher concentration of lidocaine was required than the concentration needed to block the effects of cues presented alone. The reasons why a $10 \mu \mathrm{g}$ lidocaine dose was ineffective in the cocaine priming tests are not entirely clear. The data in Table 1 suggest that differences in the time course of inactivation for 10,56 , and $100 \mu \mathrm{g}$ doses cannot explain the ineffectiveness of $10 \mu \mathrm{g}$ during cocaine priming tests because the time course for each dose was similar. The only difference between the two types of reinstatement tests was the injection of $20 \mathrm{mg} / \mathrm{kg}$ cocaine $25 \mathrm{~min}$ before lidocaine infusion

\section{Food-Maintained Responding}

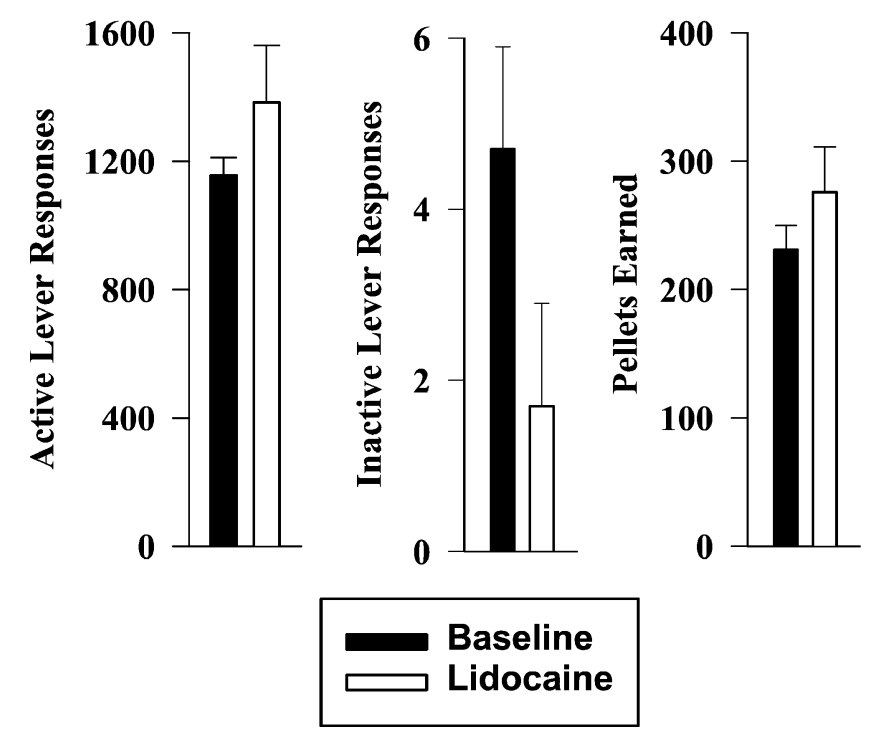

Figure 8. Food-maintained responding after lidocaine inactivation of the basolateral amygdala $(n=3)$. Active lever responses are shown on the left, inactive lever responses in the middle, and number of pellets earned on the right. Values are the mean \pm SEM.

during the priming tests. Although cocaine has an approximately fivefold greater affinity for the sodium channel than lidocaine in rat brain (Wilcox et al., 2001), previous studies have shown competitive antagonism between cocaine and lidocaine at a single sodium channel site during stimulation of superfused tissue (Liu et al., 1996). It is thought that the fast on-off sodium channelbinding properties of lidocaine offset the slow on-off kinetics of cocaine to produce competitive antagonism of cocaine (Winecoff et al., 1994). Given their fivefold different affinities for the sodium channel, at least a fivefold higher concentration of locally applied lidocaine might be required to inactivate both myelinated and nonmyelinated components of neurons in the rBLA and cBLA when cocaine is present than when it is not.

\section{Basis of dissociable effects}

One explanation for the functional heterogeneity between the rBLA and cBLA in regulating drug-seeking behavior under maintenance and reinstatement conditions may be related to their connections with segregated, but parallel, corticostriatalpallidothalamic circuits (Gröenewegen et al., 1991; Shinonaga et al., 1994; Wright and Gröenewegen, 1996). The rBLA is connected in series with the dorsal agranular insular region of the lateral prefrontal cortex and the intermediodorsal thalamic nucleus. Each site in this loop predominantly innervates the lateral nucleus accumbens (core subregion). The cBLA on the other hand, is connected in series with the prelimbic region of the medial prefrontal cortex and paraventricular thalamic nucleus. Each of these sites predominantly innervates the medial nucleus accumbens (medial shell subregion). Although the specific functions of these two loops are primarily unknown, studies have shown that the core and medial shell subregions of the nucleus accumbens are functionally distinct with regard to the regulation of rewardrelevant behavior and discriminability of conditioned stimulus cues (Parkinson et al., 1999; Ito et al., 2000). The nucleus accumbens core and shell, in turn, have differential connections to 
distinct subregions of the ventral pallidum and substantia nigra (Gröenewegen et al., 1990; 1991). Other distinctions between the rBLA and cBLA that may be relevant for dissociable functioning are their differences in basal levels of dopamine and numbers of tyrosine hydroxylase-containing neurons (Young and Rees, 1998; Brinley-Reed and McDonald, 1999).

\section{Drug-seeking versus drug-taking behavior}

Although it is clear that the rBLA and cBLA may dissociably regulate drug-seeking behavior during maintenance and reinstatement, sites outside the basolateral amygdala must regulate drug-taking behavior. This conclusion is derived from the fact that this aspect of behavior was not altered by lidocaine inactivation of either the rBLA or cBLA. Whether drug-taking behavior involves regulation by other memory systems is not entirely clear at this time. However, given rats maintained enough responses after lidocaine infusions to keep drug-taking behavior constant as drug-seeking behavior decreased suggests that a conditioning process may be involved. One possibility is that contextual cues in the self-administration environment served to maintain drug-taking behavior, suggesting possible regulation of drugtaking behavior by a hippocampal-dependent memory system that, among other things, mediates associations between stimuli (Eichenbaum, 1997). Lesions to the ventral subiculum of the hippocampus have been shown to impair approach to a sucrosepaired stimulus and to blunt the stimulant effects of D-amphetamine (Burns et al., 1993). Based on these and other studies, it has been suggested that a function of the ventral subiculum is to determine reward efficacy (Hitchcott and Phillips, 1997), which in turn may help to regulate drug intake. We have unpublished preliminary data showing that bilateral inactivation of the dorsal subiculum with $100 \mu \mathrm{g}$ of lidocaine moderately, but significantly, reduced drug-taking behavior without altering drugseeking behavior when maintained by a second-order schedule of cocaine delivery. In addition, lesions of either the dorsal or ventral subiculum have been shown to moderately impair acquisition criteria performance during the acquisition phase of cocaine self-administration studied under a FR-1 schedule of drug delivery (Caine et al., 2001).

\section{Conclusions}

Using a second-order schedule of cocaine delivery, dissociable control over drug-seeking behavior by the rBLA and cBLA was demonstrated. These findings extend previous investigations of the basolateral amygdala (Whitelaw et al., 1996; Meil and See, 1997; Grim and See, 2000; Weiss et al., 2000) to suggest that the neural circuitry within the basolateral amygdala for regulating drug-seeking behavior is more functionally heterogeneous than commonly thought. This heterogeneity might arise from their distinct neuroanatomical connections within corticostriatalpallidothalamic circuits. The role of other memory systems in regulating addiction-related behavior is a current issue (for review, see Everitt and Robbins, 2000). Findings from present and past studies indicate that the same associative functions that maintain good mental health can also contribute to maintain a brain disease, namely drug addiction and its relapse. How to maintain normal memory system function while eliminating abnormal aspects in the cocaine-addicted brain will become a challenge as novel treatment strategies evolve.

\section{REFERENCES}

Breiter HC, Gollub RL, Weisskoff RM, Kennedy DN, Makris N, Berke JD, Goodman JM, Kantor HL, Gasrfriend DR, Riorden JP, Mathew
RT, Rosen BR, Hyman SE (1997) Acute effects of cocaine on human brain activity and emotion. Neuron 19:591-611.

Brinley-Reed M, McDonald AJ (1999) Evidence that dopaminergic axons provide a dense innervation of specific neuronal subpopulations in the rat basolateral amygdala. Brain Res 850:127-135.

Burns L, Robbins T, Everitt B (1993) Differential effects of excitotoxic lesions of the basolateral amygdala, ventral subiculum and medial prefrontal cortex on responding with conditioned reinforcement and locomotor activity potentiated by intra-accumbens infusions of d-amphetamine. Behav Brain Res 55:167-183.

Cador M, Robbins TW, Everitt BJ (1989) Involvement of the amygdala in stimulus-reward associations: Interactions with the ventral striatum. Neuroscience 30:77-86.

Caine SB, Humby T, Robbins TW, Everitt BJ (2001) Behavioral effects of psychomotor stimulants in rats with dorsal or ventral subiculum lesions: locomotion, cocaine self-administration, and prepulse inhibition of startle. Behav Neurosci 115:880-894.

Childress AR, Mozley PD, McElgin W, Fitzgerald J, Reivich M, O'Brien CP (1999) Limbic activation during cue-induced cocaine craving. Am J Psychiat 156:11-18.

Eichenbaum H (1997) Declarative memory: insights from cognitive neurobiology. Annu Rev Psychol 48:547-572.

Everitt BJ, Robbins TW (2000) Second-order schedules of drug reinforcement in rats and monkeys: measurement of reinforcing efficacy and drug-seeking behaviour. Psychopharmacology 153:17-30.

Everitt BJ, Morris KA, O'Brien A, Robbins TW (1991) The basolatera amygdala-ventral striatal system and conditioned place preference: further evidence of limbic-striatal interactions underlying rewardrelated processes. Neuroscience 42:1-18.

Goldberg S, Kelleher R, Goldberg D (1981) Fixed-ratio responding under second-order schedules of food presentation or cocaine injection. J Pharmacol Exp Ther 218:271-281.

Grant S, London E, Newlin D, Villemagne V, Liu X, Contoreggi C, Phillips R, Kimes A, Margolin A (1996) Activation of memory circuits during cue-elicited cocaine craving. Proc Natl Acad Sci USA 93:12040-12045.

Grim JW, See RE (2000) Dissociation of primary and secondary rewardrelevant limbic nuclei in an animal model of relapse. Neuropsychopharmacology 22:473-479.

Gröenewegen H, Berendse H, Wolters J, Lohman A (1990) The anatomical relationship of the prefrontal cortex with the striatopallidal system, the thalamus and the amygdala: evidence for a parallel organization. Prog Brain Res 85:95-118.

Gröenewegen H, Berendse H, Meredith G, Haber S, Voorn P, Wolters J, Lohman A (1991) Functional anatomy of the ventral, limbic systeminnervated striatum. In: The mesolimbic dopamine system: from motivation to action (Willner P, Scheel-Kruger J, eds), pp 19-59. New York: Wiley.

Hitchcott PK, Phillips GD (1997) Amygdala and hippocampus control dissociable aspects of drug-associated conditioned rewards. Psychopharmacology 131:187-195.

Ito R, Dalley JW, Howes SR, Robbins TW, Everitt BJ (2000) Dissociation in conditioned dopamine release in the nucleus accumbens core and shell in response to cocaine cues and during cocaine-seeking behavior in rats. J Neurosci 20:7489-7495.

Kantak KM, Collins SL, Lipman EG, Bond J, Giovanoni K, Fox BS (2000) Evaluation of anti-cocaine antibodies and a cocaine vaccine in a rat self-administration model. Psychopharmacology 148:251-262.

Kantak KM, Green-Jordan K, Valencia E, Kremin T, Eichenbaum HB (2001) Cognitive task performance following lidocaine-induced inactivation of different sites within the basolateral amygdala and dorsal striatum. Behav Neurosci 115:589-601.

Liu D, Hariman RJ, Bauman JL (1996) Cocaine concentration-effect relationship in the presence and absence of lidocaine: evidence of competitive binding between cocaine and lidocaine. J Pharmacol Exp Ther 276:568-577.

Lomber SG (1999) The advantages and limitations of permanent or reversible deactivation techniques in the assessment of neural function. J Neurosci Methods 86:109-117.

Maas LC, Lukas SE, Kaufman MJ, Weiss RD, Daniels SL, Rogers VW, Kukes TJ, Renshaw PF (1998) Functional magnetic resonance imaging of human brain activation during cue-induced cocaine craving. Am J Psychiat 155:124-126.

Malpeli JG (1999) Reversible inactivation of subcortical sites by drug injection, J Neurosci Methods 86: 119-128.

Mantsch JR, Goeders NE (1999) Ketoconazole does not block cocaine discrimination or the cocaine-induced reinstatement of cocaineseeking behavior. Pharmacol Biochem Behav 64:65-73.

Markou A, Arroyo M, Everitt BJ (1999) Effects of contingent and noncontingent cocaine on drug-seeking behavior measured using a secondorder schedule of cocaine reinforcement in rats. Neuropsychopharmacology 20:542-555.

Martin JH (1991) Autoradiographic estimation of the extent of reversible inactivation produced by microinjection of lidocaine and muscimol in the rat. Neurosci Lett 127:160-164. 
Martin JH, Ghez C (1999) Pharmacological inactivation in the analysis of the central control of movement. J Neurosci Methods 86:145-159.

Meil WM, See RE (1997) Lesions of the basolateral amygdala abolish the ability of drug associated cues to reinstate responding during the withdrawal from self-administered cocaine. Behav Brain Res 87:139-148.

Mutschler NH, Miczek KA (1998) Withdrawal from IV cocaine "binges" in rats: ultrasonic distress calls and startle. Psychopharmacology 135:161-168.

Olmstead MC, Parkinson JA, Miles FJ, Everitt BJ, Dickinson A (2000) Cocaine-seeking by rats: regulation, reinforcement, activation Psychopharmacology 152:123-131.

Parkinson JA, Olmstead MC, Burns LH, Robbins TW, Everitt BJ (1999) Dissociation in effects of lesions of $t$ he nucleus accumbens core and shell on appetitive pavlovian approach behavior and the potentiation of conditioned reinforcement and locomotor activity by $D$-amphetamine. J Neurosci 19:2401-2411.

Sandkühler J, Gebhart GF (1984) Relative contributions of the nucleus raphe magnus and adjacent medullary reticular formation to the inhibition by stimulation in the periaqueductal gray of a spinal nociceptive reflex in pentobarbital-anesthetized rat. Brain Res 305:77-87.

Sandkühler J, Maisch B, Zimmermann M (1987) The use of local anaesthetic microinjections to identify central pathways: a quantitative evaluation of the time course and extent of the neuronal block. Exp Brain Res 68:168-178.

Schmidt EF, Sutton MA, Schad CA, Karanian DA, Brodkin ES, Self DW (2001) Extinction training regulates tyrosine hydroxylase during withdrawal from cocaine self-administration. J Neurosci 21:RC137.

See RE, Grimm JW, Kruzich PJ, Rustay N (1999) The importance of a compound stimulus in conditioned drug-seeking behavior following one week of extinction from self-administered cocaine in rats. Drug Alcohol Depend 57:41-49.

Shinonaga Y, Takada M, Mizuno N (1994) Topographic organization of collateral projections from the basolateral amygdaloid nucleus to both the prefrontal cortex and nucleus accumbens in the rat. Neuroscience 58:389-397.

Spealman RD, Barrett-Larimore RL, Rowlett JK, Platt DM, Khroyan TV (1999) Pharmacological and environmental determinants of re- lapse to cocaine-seeking behavior. Pharmacol Biochem Behav 64:327-336.

Swanson L (1992) Brain maps: structure of the rat brain. Amsterdam: Elsevier.

Tehovnik EJ, Sommer MA (1997) Effective spread and timecourse of neural inactivation caused by lidocaine injection in monkey cerebral cortex. J Neurosci Methods 74:17-26.

Tran-Nguyen LTL, Fuchs RA, Coffey GP, Baker DA, O'Dell LE, Neisewander JL (1998) Time-dependent changes in cocaine-seeking behavior and extracellular dopamine levels in the amygdala during cocaine withdrawal. Neuropsychopharmacology 19:48-59.

Wang GJ, Volkow ND, Fowler JS, Cervany P, Hitzemann RJ, Pappas NR, Wong CT, Felder C (1999) Regional brain metabolic activation during craving elicited by recall of previous drug experiences. Life Sci 64:775-784.

Weiss F, Maldonado CS, Parsons LH, Kerr TM, Smith DL, Ben-Shahar $\mathrm{O}$ (2000) Control of cocaine-seeking behavior by drug-associated stimuli in rats: effects on recovery of extinguished operant-responding and extracellular dopamine levels in amygdala and nucleus accumbens. Proc Natl Acad Sci USA 97:4321-4326.

Whitelaw R, Markou A, Robbins TW, Everitt BJ (1996) Excitotoxic lesions of the basolateral amygdala impair the acquisition of cocaineseeking behaviour under a second-order schedule of reinforcement. Psychopharmacology 127:213-224.

Wilcox KM, Paul IA, Ordway GA, Woolverton WL (2001) Role of dopamine transporter and the sodium channel in the cocaine-like discriminative stimulus effects of local anesthetics in rats. Psychopharmacology 157:260-268.

Winecoff AP, Hariman RJ, Grawe JJ, Wang Y, Bauman JL (1994) Reversal of the electrocardiographic effects of cocaine by lidocaine. Part 1. Comparison with sodium bicarbonate and quinidine. Pharmacotherapy 14:698-703.

Wright CI, Gröenewegen HJ (1996) Patterns of overlap and segregation between insular cortical, intermediodorsal thalamic and basal amygdaloid afferents in the nucleus accumbens of the rat. Neuroscience 73:359-373.

Young AM, Rees KR (1998) Dopamine release in the amygdaloid complex of the rat, studied by brain microdialysis. Neurosci Lett 249:49-52. 